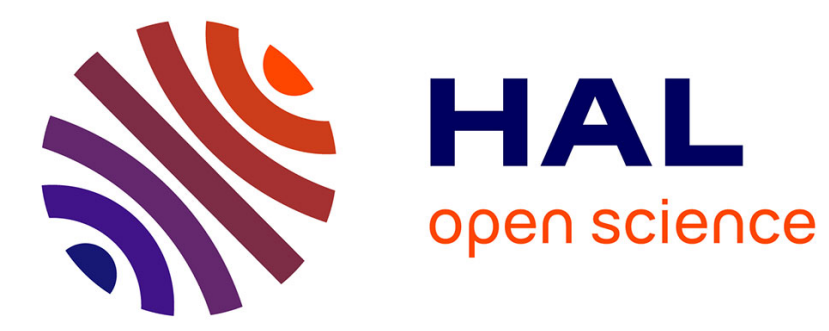

\title{
A passive electric controller for multimodal vibrations of thin plates
}

Silvio Alessandroni, Ugo Andreaus, Francesco Dell'Isola, Maurizio Porfiri

\section{To cite this version:}

Silvio Alessandroni, Ugo Andreaus, Francesco Dell'Isola, Maurizio Porfiri. A passive electric controller for multimodal vibrations of thin plates. Computers \& Structures, 2005, pp.15. hal-00498461

\section{HAL Id: hal-00498461 \\ https://hal.science/hal-00498461}

Submitted on 7 Jul 2010

HAL is a multi-disciplinary open access archive for the deposit and dissemination of scientific research documents, whether they are published or not. The documents may come from teaching and research institutions in France or abroad, or from public or private research centers.
L'archive ouverte pluridisciplinaire HAL, est destinée au dépôt et à la diffusion de documents scientifiques de niveau recherche, publiés ou non, émanant des établissements d'enseignement et de recherche français ou étrangers, des laboratoires publics ou privés. 


\title{
A passive electric controller for multimodal vibrations of thin plates
}

\author{
S. Alessandroni ${ }^{\text {a }}$, U. Andreaus ${ }^{\text {a }}$, F. dell'Isola ${ }^{\text {a,* }}$, M. Porfiri ${ }^{a, b}$ \\ a Dipartimento di Ingegneria Strutturale e Geotecnica, Università di Roma La Sapienza, 00184 Roma, Italia \\ ${ }^{\mathrm{b}}$ Department of Engineering Science and Mechanics, Virginia Tech, Blacksburg, VA 24061, USA
}

Accepted 26 August 2004

Available online 26 February 2005

\begin{abstract}
A dynamic passive controller for thin plate vibrations is here presented. The vibrations damping is obtained by uniformly distributing an array of piezoelectric elements on the host plate, and by interconnecting their terminals via a passive electric circuit.

The use of an electric network having the same structure as the plate equations (i.e., plate analog network) assures a multiresonant and broad band electromechanical coupling. The governing equations of the proposed network are derived by paralleling the Lagrangian functional of a discretized Kirchhoff-Love plate with that of a lumped, lossless and reciprocal circuit. A possible realization of the plate analog network is also proposed under the form of a circuit constituted by capacitors, inductors and transformers only. The appropriate insertion of optimized resistors in the found analog circuit allows for effecting multimodal damping of forced oscillations.

The efficiency of the proposed strategy is validated through the analysis of a realistic simply supported plate. Exact solutions are computed for interesting mechanical disturbances.
\end{abstract}

(c) 2005 Elsevier Ltd. All rights reserved.

Keywords: Smart structures; Plates; Piezoelectric transducers; Distributed control

\section{Introduction}

The increasing interest in lightweight structures has given new stimuli in the vibrations control research field. Indeed, lightweight structures are inclined to undergo

\footnotetext{
* Corresponding author. Tel.: +39064 45845397; fax: +39064 884852.

E-mail addresses: salessan@vt.edu (S. Alessandroni), ugo. andreaus@uniroma1.it (U. Andreaus), francesco.dellisola@ uniroma1.it (F. dell'Isola), maurizio.porfiri@uniroma1.it (M. Porfiri).
}

excessive vibrations which limit the structural performances and accelerate the degradation processes. Piezoelectric transducers in conjunction with appropriate control circuits represent successful and widespread devices for structural vibrations suppression.

During the last two decades, several control strategies based on piezoelectric ceramics together with electric circuits have been proposed. In the so-called "electronic damping" (see, e.g., [11,14]) a set of piezoelectric devices is placed on a host structure to simultaneously sense and control mechanical oscillations. The deformation of the sensors results in electrical signals, which are conditioned by suitably-designed feedback

0045-7949/\$ - see front matter (C) 2005 Elsevier Ltd. All rights reserved. doi:10.1016/j.compstruc.2004.08.028 
electronics and then applied to the actuators. In this framework, the piezoelectric transducers' driving requires complex power amplifiers and associated precise sensing electronics. Furthermore, the presence of an active controller can induce instability in the closed-loop system. Spillover phenomena can also be introduced, inducing dramatic oscillations of the structure at high frequencies. The "electronic damping" may also be realized (as experimentally shown in [13]) by a digital controller, using a computer and an analog digital conversion card.

An interesting development of "electronic damping" has been proposed in $[3,15,19]$, where the design of optimal distributed electronic active controllers is addressed. The approach outlined in [3] includes a distributed array of piezoelectric elements periodically positioned over a host structure and a distributed interconnecting (active) electronic circuit. The distributed sensors and actuators are employed to sense the deformation of the flexible structure and to exert control actions at every location, respectively. The purpose of the distributed electronic circuit is to extract the complete state of the plant from the sensors, to optimally condition these signals, and to feed the actuators at high voltages. The resulting smart structure is able to efficiently suppress mechanical vibrations induced by broadband disturbances. Nevertheless, the intrinsic active nature of the controller and the complexity of the required circuitry may limit its technical feasibility and exploitation in industrial applications.

In [12] the possibility of damping mechanical vibrations by means of a single piezoelectric transducer positioned on a structural element and shunted with completely passive electrical circuits is investigated. If the piezoelectric element is shunted with an $R L$ impedance, the passive network combined with the inherent capacitance of the piezoelectric transducer behaves as a vibration absorber, and induces a damped electromechanical beating. The resonance can be tuned so that the piezoelectric element acts as a vibration absorber. An efficient single-mode control of structural vibrations is achieved whenever the resonant circuit is tuned to the mechanical mode to be suppressed. Nevertheless, the efficiency of the electromechanical coupling strongly depends on the position of the transducer over the host structure. Moreover, the technical feasibility of the passive piezoelectric controller proposed in [12] is limited, since unrealistically large inductances are required to produce low-frequency electrical resonance with the small inherent capacitance of the piezoelectric transducer.

In order to overcome these drawbacks without compromising the advantages featured by passive control the concept of Piezo-ElectroMechanical (PEM) structures has been introduced (see, e.g., [7,2,21]). Within this framework it is proposed to position a distributed array of piezoelectric elements on a host structure and to inter- connect their electric terminals via an appropriate distributed passive electric circuit. In [26] it is shown that the maximum energy transfer rate between the mechanical and electrical subsystems is obtained by exploiting analog networks (in the sense specified e.g., in [5]). Indeed, when the electric controller is governed by the same equations of the mechanical structure, a multiresonant coupling is accomplished. Furthermore, the similarity in between the mechanical and electric modal shapes assures a substantial energy transfer for any initial condition. Nevertheless, the problem of finding technologically convenient realizations of analog networks for common structural elements is often very complicated. Similar synthesis problems have been addressed during the fifties dealing with the so-called analog computers (see, e.g., [20,23]).

A first archetype of a PEM plate, using a suboptimal electric controller can be found in [27], where it is proposed to interconnect the electric terminals of each pair of adjacent transducers via a floating $R L$ impedance. The main advantage of this strategy is the drastic reduction of employed optimal inductances, thus making conceivable the realization of a truly passive network. Nevertheless a multimodal control is not yet guaranteed and it is only possible to effectively damp only one mode.

In [1] it is shown that by using negative inductors it is possible to realize an analog circuit of the KirchhoffLove $(\mathrm{K}-\mathrm{L})$ plate. Nevertheless, negative inductors are active elements and have to be simulated by electronic circuits.

In the present paper we address the following (interrelated) problems:

1. synthesizing an analog circuit of a $\mathrm{K}-\mathrm{L}$ plate constituted solely by passive elements;

2. modelling the piezo-composite thin plate, comprised of the given host plate and the array of periodically distributed piezoelectric elements;

3. modelling the Piezo-ElectroMechanical plate constituted by the piezo-composite plate coupled to the analog circuit;

4. analyzing the free vibrations of non-dissipative simply supported PEM plates;

5. completing the electric circuit with suitable resistors in order to assure a broadband suppression of forced oscillations;

6. designing a realistic PEM plate and studying its behavior when excited by external disturbances.

\section{Synthesis of a completely passive circuit analog to a Kirchhoff-Love plate}

In this section we design a lumped electric circuit analog to a Kirchhoff-Love plate which 
- is governed by finite dimensional Lagrange equations;

- is constituted by completely passive elements, i.e., circuits in which no external feeding is needed;

- can be tuned to the structural vibration frequencies using very low (and therefore technically realizable) nominal impedances.

To our knowledge a circuit with the listed features is not available in the literature. We underline that such a circuit represents an analog computer applicable for studying thin plate vibrations.

\subsection{Lagrange functional governing the motion of $K-L$ plates}

Let us consider a homogeneous square $\mathrm{K}-\mathrm{L}$ plate, the vibration of which is governed (see, e.g., [10]) by a Lagrange functional

$\mathscr{L}^{m}=T^{m}-U^{m}$

defined on a set of admissible displacement fields. The strain and kinetic energies appearing in (1) are, respectively, given by

$$
\begin{aligned}
U^{m}= & \frac{S}{2} \int_{\Omega}\left(\left(\partial_{11} u\right)^{2}+\left(\partial_{22} u\right)^{2}+2 v\left(\partial_{11} u\right)\left(\partial_{22} u\right)\right. \\
& \left.+2(1-v)\left(\partial_{12} u\right)^{2}\right) \mathrm{d} \Omega,
\end{aligned}
$$

and

$T^{m}=\frac{\rho h}{2} \int_{\Omega} \dot{u}^{2} \mathrm{~d} \Omega$

The meanings of the introduced symbols are $S=\frac{h^{3} E}{12\left(1-v^{2}\right)}$, bending stiffness; $h$, thickness; $E$, Young's modulus; $v$, Poisson ratio; $\Omega$, plate domain; $u$, mid-surface deflection; $\rho$, mass density per unit volume; superimposed dot, derivative with respect to the time $t$. Moreover, we have introduced a system of Cartesian coordinates $\left(x_{1}, x_{2}\right)$ on the plate reference configuration $\Omega$ and denoted by $\partial_{1}, \partial_{2}$ the derivatives with respect to $x_{1}, x_{2}$ variables, respectively.

The $\mathrm{K}-\mathrm{L}$ plate is an infinite dimensional mathematical model. In order to synthesize one among its lumped circuital analogs we introduce a finite set of Lagrange parameters describing - in an approximate way-the state of the plate: the set of deflections sampled at fixed grid points in the plate reference configuration. Let us label the generic point of the grid by the subscripts ()$_{i, j}$, so that the value at this point of a generic scalar field $f$ will be denoted by $f_{i, j}$. The step of the grid (assumed to be equal along both $x_{1}$ and $x_{2}$ directions) will be denoted by $\varepsilon$. Consistently, the spatial derivatives of the field $f$ will be approximated by the following finite differences operators:

$$
\begin{aligned}
\left(\partial_{1} f\right)_{i, j} & \approx \frac{1}{\varepsilon}\left(\Delta_{1} f\right)_{i, j}=\frac{f_{i+1, j}-f_{i, j}}{\varepsilon}, \\
\left(\partial_{2} f\right)_{i, j} & \approx \frac{1}{\varepsilon}\left(\Delta_{2} f\right)_{i, j}=\frac{f_{i, j+1}-f_{i, j}}{\varepsilon} \\
\left(\partial_{11} f\right)_{i, j} & \approx \frac{1}{\varepsilon^{2}}\left(\Delta_{11} f\right)_{i, j}=\frac{f_{i+1, j}-2 f_{i, j}+f_{i-1, j}}{\varepsilon^{2}}, \\
\left(\partial_{22} f\right)_{i, j} & \approx \frac{1}{\varepsilon^{2}}\left(\Delta_{22} f\right)_{i, j}=\frac{f_{i, j+1}-2 f_{i, j}+f_{i, j-1}}{\varepsilon^{2}}, \\
\left(\partial_{12} f\right)_{i, j} & \approx \frac{1}{\varepsilon^{2}}\left(\Delta_{12} f\right)_{i, j}=\frac{\left(f_{i+1, j+1}-f_{i, j+1}\right)-\left(f_{i+1, j}-f_{i, j}\right)}{\varepsilon^{2}} .
\end{aligned}
$$

Forward differences are applied when approximating differential operators which present odd differentiationorders with respect to one variable, while centered finite differences are used when even differentiation-orders are present. Obviously, different choices are possible each one leading to different analog circuital topologies. We explicitly remark that the chosen discretization scheme, with an extremely course "stencil" (see, e.g., [24]), will lead to a simple electric analog circuit.

Next, we discretize the $\mathrm{K}-\mathrm{L}$ plate Lagrange functional by expressing (1) by suitable Riemann sums. Therefore, the discretized deformation energy $U_{d}^{m}$ and kinetic energy $T_{d}^{m}$ of the $\mathrm{K}-\mathrm{L}$ plate, can be expressed by

$$
\begin{aligned}
U_{d}^{m}= & \frac{S}{2 \varepsilon^{2}} \sum_{i, j}\left[\left(\Delta_{11} u\right)_{i, j}^{2}+\left(\Delta_{22} u\right)_{i, j}^{2}+2 v\left(\Delta_{11} u\right)_{i, j}\left(\Delta_{22} u\right)_{i, j}\right. \\
& \left.+2(1-v)\left(\Delta_{12} u\right)_{i, j}^{2}\right] \\
T_{d}^{m}= & \frac{\rho h}{2} \varepsilon^{2} \sum_{i, j}(\dot{u})_{i, j}^{2} .
\end{aligned}
$$

The approximating discrete Lagrangian system that we consider is close to the $\mathrm{K}-\mathrm{L}$ plate model like the discrete differences are close to the derivatives: the Lagrange functionals considered in both models are likely to be sufficiently close when suitable regularity conditions on displacement fields are assumed. We leave to a more accurate mathematical treatment a precise statement of such regularity conditions and error estimates (see, e.g., [24]).

\subsection{Electric analog}

It is well known (see, e.g., [5]) that for any given quadratic Lagrangian, say

$\mathscr{L}_{d}^{m}:=T_{d}^{m}-U_{d}^{m}$,

it is possible to synthesize a lumped circuit governed by it. This can be done at least in two different ways:

1. paralleling generalized displacements with fluxlinkages, 


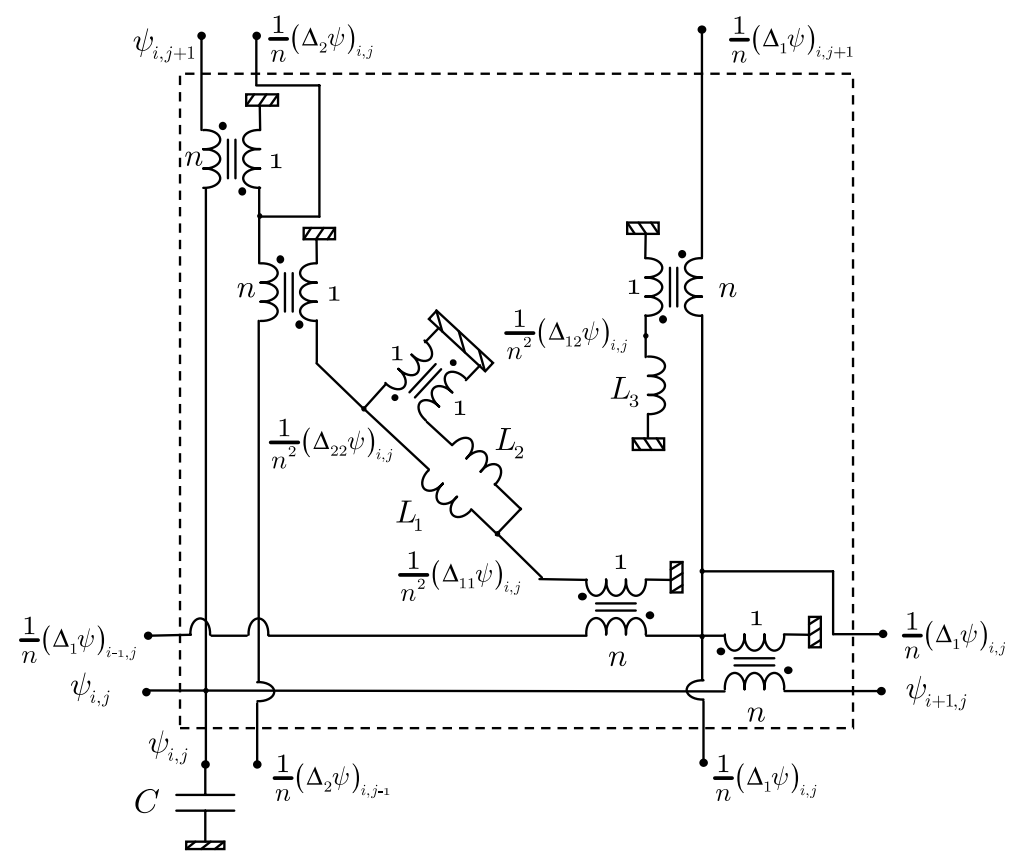

Fig. 1. Elementary module of the circuit analog to the K-L plate.

2. paralleling generalized displacements with stored charges.

When the first (second) analogy is considered, kinetic (strain) energy is associated with electric (magnetic) energy and strain (kinetic) energy is paralleled by magnetic (electric) energy. In what follows we will refer to the first option. Indeed, we will associate to every node of the sampling grid a so-called principal node (denoted by $n_{i, j}$ ) of the analog circuit. The timeintegral $\psi_{i, j}$ of its potential drop with respect to ground (i.e., the flux-linkage) will correspond to the deflection $u_{i, j}$. By noticing that the kinetic energy $T_{d}^{m}$ in (3) $)_{2}$ depends only on the sampled velocities, it is easy to see that an array of equal capacitors, connecting each principal node to ground, provides the needed electric energy. Consequently, the elastic energy $U_{d}^{m}$ in (3) (including differences among sampled displacements) may be paralleled by the magnetic energy $U_{d}^{e}$ stored into a multiterminal network, constituted by a set of inductors and transformers, interconnecting the principal nodes. Transformers do not store energy, they simply connect the nodes $n_{i, j}$ to some suitable extra auxiliary ones. The flux-linkages of these auxiliary nodes are expressed in terms of the flux-linkages $\psi_{i, j}$ by means of exactly those finite differences determining the dependence of $U_{d}^{m}$ on the deflections $u_{i, j}$.

Because of the existing analogy, the synthesized electric circuit will be coupled to the plate only in dynamic conditions: it is an open problem to determine a static analog for $\mathrm{K}-\mathrm{L}$ plate. ${ }^{1}$

In Fig. 1 it is presented one of the possible topologies for the aforementioned multiterminal network (corresponding to the $i, j$ node) capable of realizing an electric analog of the $\mathrm{K}-\mathrm{L}$ plate. In Fig. 2 the connection among different modules is shown.

Let us try to give, in few steps, a brief explanation of the proposed analog circuit, remarking the role played by each electric component:

1. The electric energy stored in a generic capacitor $C$ (see Fig. 3) depends quadratically on the voltage across its terminals as follows:

$T^{e}=\frac{1}{2} C\left(\dot{\psi}_{a}-\dot{\psi}_{b}\right)^{2}$

Since the capacitor in Fig. 1 connects $n_{i, j}$ to ground (i.e., $\psi_{a}=\psi_{i, j}, \psi_{b}=0$ ) the total electric energy due to the entire analog circuit is

$T_{d}^{e}=\frac{C}{2} \sum_{i, j}(\dot{\psi})_{i, j}^{2}$,

which is analog to the kinetic energy in $(3)_{2}$.

\footnotetext{
${ }^{1}$ The difficulties in finding a static analog for KL plate resides in the impossibility of realizing a transformer in DC (direct current).
} 


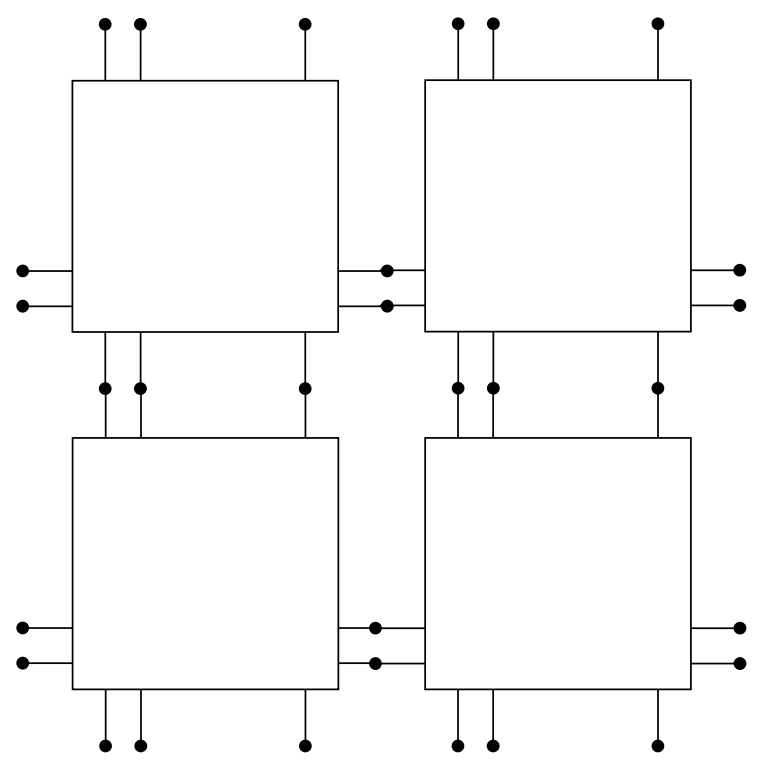

Fig. 2. Sketch of the connection topology among the analog circuital modules.
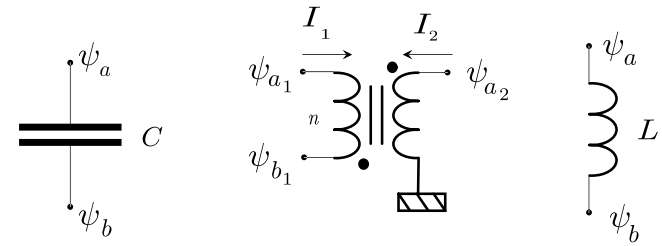

Fig. 3. Electric components used in the analog circuit: capacitor, transformer, inductor.

2. An electric transformer does not stores energy, and it simply imposes a linear constraint among the fluxlinkages at its terminals (referring in particular to the configuration in Fig. 3): ${ }^{2}$

$\psi_{a_{2}}=\frac{\psi_{b_{1}}-\psi_{a_{1}}}{n}$.

The two transformers interconnecting $n_{i, j}$ to $n_{i+1, j}$ and $n_{i, j+1}$ in Fig. 1 provide auxiliary nodes having flux-linkages proportional to the first-order finite differences: $\left(\Delta_{1} \psi\right)_{i, j} / n$ and $\left(\Delta_{2} \psi\right)_{i, j} / n$. The other three

\footnotetext{
absorption

$I_{1}\left(\dot{\psi}_{a_{1}}-\dot{\psi}_{b_{1}}\right)+I_{2} \dot{\psi}_{a_{2}}=0$,

it follows that

$-n I_{1}+I_{2}=0$.
}

${ }^{2}$ Let us explicitly remark that, by denoting with $I_{1}$ and $I_{2}$ the currents flowing through the transformers ports, by using the constraint (5) and by imposing the condition of no-energy- transformers interconnect these auxiliary nodes to provide further nodes exhibiting flux-linkages proportional to the second-order differences: $\left(\Delta_{11} \psi\right)_{i, j} /$ $n^{2},\left(\Delta_{22} \psi\right)_{i, j} / n^{2},\left(\Delta_{12} \psi\right)_{i, j} / n^{2}$. These last nodes may be properly interconnected to inductors storing the magnetic energies analog to the strain energy contributions appearing in (3) .

3. The magnetic energy stored in an inductor $L$ depends quadratically on the flux-linkage difference across its terminals (see again Fig. 3):

$U^{e}=\frac{1}{2 L}\left(\psi_{a}-\psi_{b}\right)^{2}$,

hence it is easy to see that the inductors $L_{1}, L_{2}$ and $L_{3}$ in Fig. 1 store the following amounts of magnetic energy:

$$
\begin{aligned}
U_{1}^{e} & =\frac{1}{2 L_{1} n^{4}}\left(\left(\Delta_{11} \psi\right)_{i, j}-\left(\Delta_{22} \psi\right)_{i, j}\right)^{2}, \\
U_{2}^{e} & =\frac{1}{2 L_{2} n^{4}}\left(\left(\Delta_{11} \psi\right)_{i, j}+\left(\Delta_{22} \psi\right)_{i, j}\right)^{2}, \\
U_{3}^{e} & =\frac{1}{2 L_{3} n^{4}}\left(\Delta_{12} \psi\right)_{i, j}^{2} .
\end{aligned}
$$

Finally, the total magnetic energy obtained as the sum of the all contributes of each module can be rearranged as follows:

$$
\begin{aligned}
U_{d}^{e}= & \frac{1}{2 n^{4}} \frac{L_{1}+L_{2}}{L_{1} L_{2}}\left[\sum_{i, j}\left(\Delta_{11} \psi\right)_{i, j}^{2}+\left(\Delta_{22} \psi\right)_{i, j}^{2}\right. \\
& +2 \frac{L_{1}-L_{2}}{L_{1}+L_{2}}\left(\Delta_{11} \psi\right)_{i, j}\left(\Delta_{22} \psi\right)_{i, j} \\
& \left.+\frac{L_{1} L_{2}}{L_{3}\left(L_{1}+L_{2}\right)}\left(\Delta_{12} \psi\right)_{i, j}^{2}\right]
\end{aligned}
$$

which can be made analog to the deformation energy derived in $(3)_{1}$, by a proper setting of the electric parameters.

In order to derive a set of relations between the constitutive parameters of the considered plate and the nominal values of the used electric elements, we have to render the electric Lagrangian proportional to the mechanical one. ${ }^{3}$ By comparing (3) 2 with (4) and (3) with (6) we get

$\frac{1}{2 n^{4}} \frac{L_{1}+L_{2}}{L_{1} L_{2}}=k \frac{S}{2 \varepsilon^{2}}, \quad \frac{C}{2}=k \frac{\rho h}{2} \varepsilon^{2}$,
$\frac{L_{1}-L_{2}}{L_{1}+L_{2}}=v, \quad \frac{L_{2} L_{1}}{L_{3}\left(L_{1}+L_{2}\right)}=2(1-v)$,

$k$ being an arbitrary positive constant. Simple algebra allows us to get the following constraints on the electric impedances and transformers turns-ratios:

\footnotetext{
${ }^{3}$ A constant of proportionality in between the two Lagrangian guarantees that the electric and mechanical systems are governed by the same equations.
} 
$\frac{L_{1}}{L_{2}}=\frac{(1+v)}{(1-v)}, \quad \frac{L_{1}}{L_{3}}=4, \quad n^{4} L_{1} C=\frac{2 \rho h}{S(1-v)} \varepsilon^{4}$.

Once the constitutive properties of the given plate, the capacitance $C$ and the turns-ratio $n$ have been chosen, from (7) we can easily claim that the needed inductances decreases rapidly as the grid becomes more refined. Let us note that the introduction of transformers in the circuital analogs allows for the synthesis of a completely passive circuit analog (which was not possible in [1]).

\section{Modelling PEM plates}

Once the synthesis of the electric analog circuit has been worked out, the problem of using it to optimally control plate vibrations has to be tackled. To this aim, we assume that two uniform arrays of piezoelectric elements are positioned on both the surfaces of the given host plate. The dimensions of each piezoelectric patch are $l_{p} \times l_{p} \times \delta, \delta$ being the distance between the electrodes. Each piezoelectric element is poled in the transverse direction and its geometric center is coincident with the $i, j$ grid-point. The pair of elements located in correspondence of the $i, j$ node, on the upper and lower surfaces, are parallel connected to form a so called "bending" transducer, as shown in Fig. 4.

A PEM plate is conceived by substituting to the capacitor appearing at node $n_{i, j}$ of the analog circuit, the bending transducer located in correspondence of the $i, j$ grid point. This electric interconnection is due to the Norton representation of a piezoelectric element, in other words: a capacitor in parallel connection with a current generator driven by the time rate of mechanical deformation. In [27] the theoretical reasons underlying the optimality of analog circuits in multimodal vibration control are discussed. We limit ourselves here to state that an analog circuit is able to resonate at every natural frequency of the given structural element, and to exhibit exactly the same modal shapes.
In the present section, we will tackle the following problems:

1. modelling the piezo-composite thin plate, including the given host plate and the array of periodically distributed piezoelectric elements;

2. modelling the non-dissipative Piezo-ElectroMechanical plate resulting from the coupling of the piezocomposite plate and the analog circuit.

\subsection{Transducers modelling}

In the present subsection, we will derive an extremely simple model of the considered surface-bonded piezoelectric elements by assuming that the piezoelectric sheets are much thinner than the host plate $(\delta \ll h)$. Therefore, we will assume that each piezoelectric element is under plane stress conditions (along $x_{1}, x_{2}$ directions), that the electric field is constant and stays in the thickness direction, and that perfect bonding condition holds at the transducers-plate interphases. These hypotheses are well-grounded whenever negligible bonding layers can be obtained (see, e.g., [6]) and thin piezoelectrics are used (see, e.g., [25]).

Let us explicitly remark that we are interested in modelling piezoelectric transducers, i.e., simultaneously modelling sensing and actuating behaviors. Therefore, in order to get consistent theories, one has to make compatible hypotheses on the electrical and mechanical variables. For instance, when also the piezoelectric layers are assumed to undergo Kirchhoff-Love displacement fields, for consistency, the electric field cannot be regarded to be constant and much more complicated problems arise (see, e.g., [22]).

Let us consider the general three-dimensional constitutive relations for a transversely-isotropic material (Hermann-Maugin class $\infty \mathrm{mm}$, see, e.g., $[17,18]$ ), with the poling direction along the $x_{3}$ axis (thickness direction):

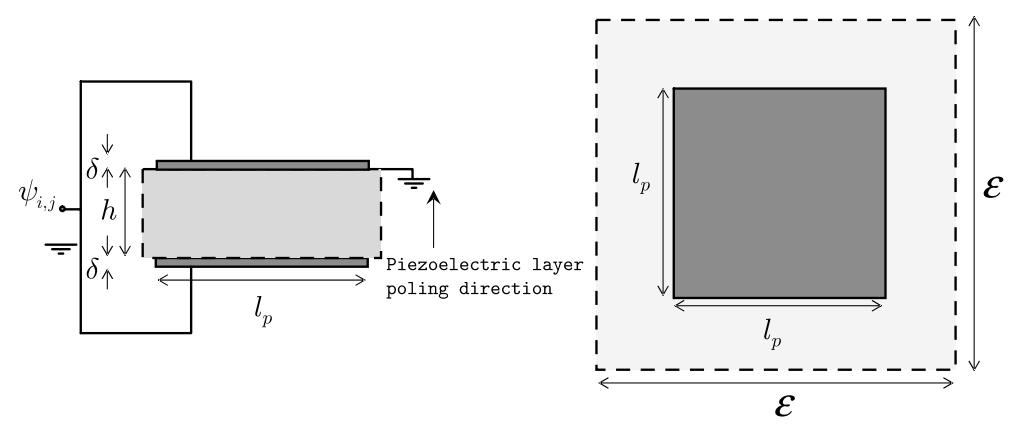

Fig. 4. Top and side views of the elementary cell of the piezo-composite plate. 


$$
\begin{aligned}
\left\{\begin{array}{c}
\varepsilon_{1} \\
\varepsilon_{2} \\
\varepsilon_{3} \\
\gamma_{23} \\
\gamma_{13} \\
\gamma_{12} \\
D_{1} \\
D_{2} \\
D_{3}
\end{array}\right\}= & {\left[\begin{array}{ccccccccc}
S_{11}^{\mathrm{E}} & S_{12}^{\mathrm{E}} & S_{13}^{\mathrm{E}} & 0 & 0 & 0 & 0 & 0 & d_{31} \\
S_{12}^{\mathrm{E}} & S_{11}^{\mathrm{E}} & S_{13}^{\mathrm{E}} & 0 & 0 & 0 & 0 & 0 & d_{31} \\
S_{13}^{\mathrm{E}} & S_{13}^{\mathrm{E}} & S_{33}^{\mathrm{E}} & 0 & 0 & 0 & 0 & 0 & d_{33} \\
0 & 0 & 0 & S_{44}^{\mathrm{E}} & 0 & 0 & 0 & d_{15} & 0 \\
0 & 0 & 0 & 0 & S_{44}^{\mathrm{E}} & 0 & d_{15} & 0 & 0 \\
0 & 0 & 0 & 0 & 0 & 2\left(S_{11}^{\mathrm{E}}-S_{12}^{\mathrm{E}}\right) & 0 & 0 & 0 \\
0 & 0 & 0 & 0 & d_{15} & 0 & e_{11}^{\mathrm{T}} & 0 & 0 \\
0 & 0 & 0 & d_{15} & 0 & 0 & 0 & e_{22}^{\mathrm{T}} & 0 \\
d_{31} & d_{31} & d_{33} & 0 & 0 & 0 & 0 & 0 & e_{33}^{\mathrm{T}}
\end{array}\right] } \\
& \left.\times \begin{array}{l}
\sigma_{1} \\
\sigma_{2} \\
\sigma_{3} \\
\tau_{23} \\
\tau_{13} \\
\tau_{12} \\
E_{1} \\
E_{2} \\
E_{3}
\end{array}\right\},
\end{aligned}
$$

where $\varepsilon_{i}$ and $\gamma_{i j}$ are the engineering strains, $D_{i}$ the electric displacement components, $\sigma_{i}$ the normal stresses, $\tau_{i j}$ the shear stresses, $E_{i}$ the electric field components, $S_{i j}^{\mathrm{E}}$ the elastic compliances, $d_{i j}$ the piezoelectric coefficients and $e_{i j}^{\mathrm{T}}$ the dielectric permittivities. In the case of plane stress (along $x_{1}, x_{2}$ directions) and electric field non-zero only in the direction $x_{3}$ of transverse isotropy the previous constitutive relations reduce to

$$
\left\{\begin{array}{c}
\varepsilon_{1} \\
\varepsilon_{2} \\
\gamma_{12} \\
D_{3}
\end{array}\right\}=\left[\begin{array}{cccc}
S_{11}^{\mathrm{E}} & S_{12}^{\mathrm{E}} & 0 & d_{31} \\
S_{12}^{\mathrm{E}} & S_{11}^{\mathrm{E}} & 0 & d_{31} \\
0 & 0 & 2\left(S_{11}^{\mathrm{E}}-S_{12}^{\mathrm{E}}\right) & 0 \\
d_{31} & d_{31} & 0 & e_{33}^{\mathrm{T}}
\end{array}\right]\left\{\begin{array}{c}
\sigma_{1} \\
\sigma_{2} \\
\tau_{12} \\
E_{3}
\end{array}\right\} .
$$

When solving for the stresses and the electric displacement, we obtain

$\left\{\begin{array}{c}\boldsymbol{\sigma} \\ D_{3}\end{array}\right\}=\left[\begin{array}{cc}\mathbf{K}_{m m} & -\mathbf{K}_{m e} \\ \mathbf{K}_{m e}^{T} & K_{e e}\end{array}\right]\left\{\begin{array}{c}\boldsymbol{\varepsilon} \\ E_{3}\end{array}\right\}$,

where $\boldsymbol{\sigma}^{T}=\left\{\begin{array}{lll}\sigma_{1} & \sigma_{2} & \tau_{12}\end{array}\right\}$ indicates the non-zero components of the stress tensor and $\boldsymbol{\varepsilon}^{T}=\left\{\begin{array}{lll}\varepsilon_{1} & \varepsilon_{2} & \gamma_{12}\end{array}\right\}$ the non-zero engineering strains (superscript $T$ denotes matrix transposition). The electromechanical "stiffness matrices" appearing in (8) are given by

$$
\begin{aligned}
& \mathbf{K}_{m m}=\left[\begin{array}{ccc}
k_{m m} & v_{p} k_{m m} & 0 \\
v_{p} k_{m m} & k_{m m} & 0 \\
0 & 0 & \frac{k_{m m}}{2}\left(1-v_{p}\right)
\end{array}\right], \quad \mathbf{K}_{m e}=k_{m e}\left\{\begin{array}{l}
1 \\
1 \\
0
\end{array}\right\}, \\
& K_{e e}=\left(e_{33}^{\mathrm{T}}-\frac{2 d_{31}^{2} E_{p}}{1-v_{p}}\right), \quad k_{m m}=\frac{E_{p}}{1-v_{p}^{2}}, \quad k_{m e}=\frac{d_{31} E_{p}}{1-v_{p}},
\end{aligned}
$$

where we have used the more familiar notation:

$S_{11}^{\mathrm{E}}=\frac{1}{E_{p}}, \quad S_{12}^{\mathrm{E}}=-\frac{v_{p}}{E_{p}}$,

involving the Young's modulus $E_{p}$ and Poisson ratio $v_{p}$.
The electric field at the $i, j$ element, assumed to be constant in the thickness direction, may be expressed in terms of the voltage, say $V_{i, j}\left(\dot{\psi}_{i, j}\right)$, by

$E_{3}=-\frac{V_{i, j}}{\delta} \operatorname{RECT}_{i, j}$

where $\operatorname{RECT}_{i, j}$ indicates the characteristic function defined by

$\operatorname{RECT}_{i, j}= \begin{cases}1, & \left|x-x_{i}\right| \leqslant l_{p} / 2, \quad \text { and } \quad\left|y-y_{j}\right| \leqslant l_{p} / 2, \\ 0, & \text { elsewhere. }\end{cases}$

The charge stored in the $i, j$ bending transducers is given by (see, e.g., [4])

$Q_{i, j}=\int_{I_{3}} \int_{\Omega} D_{3} \operatorname{RECT}_{i, j} \mathrm{~d} \Omega \mathrm{d} x_{3}$,

where

$I_{3}=\left[-\frac{h}{2}-\delta,-\frac{h}{2}\right] \cup\left[\frac{h}{2}, \frac{h}{2}+\delta\right]$.

Therefore, using (8) we obtain the following relation for the capacitance $C$ of each bending piezoelectric transducer, under no-strain conditions:

$C=2 K_{e e} \frac{l_{p}^{2}}{\delta}$.

When dealing with variational formulation for the dynamics of piezoelectric materials, several approaches may be adopted. In what follows, we will choose the displacement and the flux-linkage to be the electromechanical kinematical descriptors of the considered piezoelectric arrays (see, e.g., [9]). Therefore, the Lagrangian for the $i, j$ bending transducer, say $\left(\mathscr{L}^{p}\right)_{i, j}$, is expressed by

$\left(\mathscr{L}^{p}\right)_{i, j}=\left(T^{p}\right)_{i, j}-\left(H^{p}\right)_{i, j}$,

where $\left(T^{p}\right)_{i, j}$ denotes the kinetic energy and $\left(H^{p}\right)_{i, j}$ the enthalpy (see, e.g., [16]).

From the given assumptions on the stress and strain fields (plane stress along $x_{1}$ and $x_{2}$ and electric field in the thickness direction), electric field, and electric displacement field, the enthalpy becomes

$$
\begin{aligned}
\left(H^{p}\right)_{i, j}= & \int_{\Omega} \int_{I_{3}} \operatorname{RECT}_{i, j}\left\{\frac{1}{2} \boldsymbol{\varepsilon}^{T} \mathbf{K}_{m m} \boldsymbol{\varepsilon}-\varepsilon^{T} \mathbf{K}_{m e} E_{3}\right. \\
& \left.-\frac{1}{2} K_{e e} E_{3}^{2}\right\} \mathrm{d} x_{3} \mathrm{~d} \Omega .
\end{aligned}
$$

Let us observe that the stress fields and electric displacement can be derived from the enthalpy by

$$
\begin{aligned}
& \sigma_{1}=\frac{\partial\left(H^{p}\right)_{i, j}}{\partial \varepsilon_{1}}, \quad \sigma_{2}=\frac{\partial\left(H^{p}\right)_{i, j}}{\partial \varepsilon_{2}}, \quad \sigma_{12}=2 \frac{\partial\left(H^{p}\right)_{i, j}}{\partial \gamma_{12}}, \\
& D_{3}=-\frac{\partial\left(H^{p}\right)_{i, j}}{\partial E_{3}} .
\end{aligned}
$$


By assuming perfect bonding at the plate-transducers' interphase, it is easy to relate the kinematics of the patch $i, j$ to the kinematics of the host plate:

$\varepsilon_{1}=-\frac{h}{2} \partial_{11} u \operatorname{RECT}_{i, j} ; \quad \varepsilon_{2}=-\frac{h}{2} \partial_{22} u \operatorname{RECT}_{i, j} ;$
$\gamma_{12}=-h \partial_{12} u \operatorname{RECT}_{i, j}$.

Therefore, the enthalpy becomes

$$
\begin{aligned}
\left(H^{p}\right)_{i, j}= & \int_{\Omega} \operatorname{RECT}_{i, j}\left\{\delta ( \frac { h } { 2 } ) ^ { 2 } k _ { m m } \left[\left(\partial_{11} u\right)^{2}+\left(\partial_{22} u\right)^{2}\right.\right. \\
& \left.\left.+2 v_{p}\left(\partial_{11} u\right)\left(\partial_{22} u\right)+2\left(1-v_{p}\right)\left(\partial_{12} u\right)^{2}\right]\right\} \mathrm{d} \Omega \\
& +\int_{\Omega} \operatorname{RECT}_{i, j}\left\{h k_{m e}\left(\partial_{11} u+\partial_{22} u\right) \dot{\psi}_{i, j}-\frac{K_{e e}}{\delta} \dot{\psi}_{i, j}^{2}\right\} \mathrm{d} \Omega .
\end{aligned}
$$

On the other hand, the kinetic energy becomes

$$
\left(T^{p}\right)_{i, j}=\delta \int_{\Omega} \operatorname{RECT}_{i, j} \rho_{p} \dot{u}^{2} \mathrm{~d} \Omega
$$

where $\rho_{p}$ denotes the volumetric density of mass of the piezoelectric material.

The Lagrangian of the piezo-composite plate, say $\mathscr{L}^{p-c}$, can be expressed as the sum of (1) and the total contributions of the piezoelectric arrays, by

$$
\mathscr{L}^{p-c}=\mathscr{L}^{m}+\sum_{i, j}\left(\mathscr{L}^{p}\right)_{i, j},
$$

which yields

$$
\begin{aligned}
\mathscr{L}^{p-c}= & \frac{1}{2} \int_{\Omega}\left[\rho h+2 \rho_{p} \delta \sum_{i, j} \operatorname{RECT}_{i, j}\right] \dot{u}^{2} \mathrm{~d} \Omega \\
& -\frac{1}{2} \int_{\Omega}\left(\partial_{11} u+\partial_{22} u\right)^{2}\left[S+\delta \frac{h^{2}}{2} k_{m m} \sum_{i, j} \operatorname{RECT}_{i, j}\right] \mathrm{d} \Omega \\
& -\int_{\Omega}\left(\left(\partial_{12} u\right)^{2}-\left(\partial_{11} u\right)\left(\partial_{22} u\right)\right) \\
& \times\left[S(1-v)+\delta \frac{h^{2}}{2} k_{m m}\left(1-v_{p}\right) \sum_{i, j} \operatorname{RECT}_{i, j}\right] \mathrm{d} \Omega \\
& -\sum_{i, j} \int_{\Omega} \operatorname{RECT}_{i, j} h k_{m e}\left(\partial_{11} u+\partial_{22} u\right) \mathrm{d} \Omega \dot{\psi}_{i, j} \\
& +\frac{1}{2} C \sum_{i, j} \dot{\psi}_{i, j}^{2} .
\end{aligned}
$$

In order to be tuned to the mechanical oscillations, the analog circuit (where the set of capacitors has been substituted by the piezoelectric inherent capacitances) has to be designed to take into account the additional stiffness and mass due to the presence of the piezo-array.

\subsection{Complete Lagrangian of non-dissipative PEM plates}

In order to get some qualitative informations about PEM plates behavior, in some circumstances of interest in engineering applications, we can consider the limit for the number of transducers going to infinity. Such a formal procedure may have some direct applicative interest in vibration control of mechanical modes the wavelengths of which are much greater than the size of the transducers. In this case the homogenized model obtained after performing the aforementioned formal limit may be useful also for quantitative prevision of the behavior of PEM plates. We believe that the rigorous mathematical proof given for a similar problem in $[3,15]$ may be adapted to the case at hand. However, the homogenized model may also be of use in a preliminary dimensioning and/or as a first step in an iterative modelling procedure aiming to account more precisely for the discrete lumped nature of analog circuits.

The idea of using homogenized models instead of discrete ones is often used in mechanics and theory of circuits. Indeed, a truss modular structure and the lumped realization of a telegraphist transmission line, which are naturally described by finite dimensional Lagrangian systems, are commonly and effectively studied using PDEs obtained by suitable homogenization procedures.

It is reasonable to assume that the Lagrangian of the homogenized piezo-composite plate (10) becomes

$$
\begin{aligned}
\mathscr{L}_{\text {hom }}^{p-c}= & \frac{\rho_{t}}{2} \int_{\Omega} \dot{u}^{2} \mathrm{~d} \Omega-\frac{S_{t}}{2} \int_{\Omega}\left(\left(\partial_{11} u\right)^{2}+\left(\partial_{22} u\right)^{2}\right. \\
& \left.+2 v_{t}\left(\partial_{11} u\right)\left(\partial_{22} u\right)+2\left(1-v_{t}\right)\left(\partial_{12} u\right)^{2}\right) \mathrm{d} \Omega \\
& -g \int_{\Omega} \dot{\psi} \nabla^{2} u \mathrm{~d} \Omega+\frac{c}{2} \int_{\Omega} \dot{\psi}^{2} \mathrm{~d} \Omega,
\end{aligned}
$$

with

$$
\begin{aligned}
& \nabla^{2} u=\partial_{11} u+\partial_{22} u, \quad \rho_{t}=\rho h+2 \rho_{p} \delta \eta^{2}, \\
& \frac{1}{S_{t}}=\frac{1}{S} \eta^{2}+\frac{1}{S+\frac{\delta h^{2}}{2} k_{m m}}\left(1-\eta^{2}\right), \\
& \frac{1}{S_{t}\left(1-v_{t}\right)}=\frac{1}{S(1-v)} \eta^{2} \\
& \quad+\frac{1}{S(1-v)+\frac{\delta h^{2}}{2} k_{m m}\left(1-v_{p}\right)}\left(1-\eta^{2}\right), \\
& c=\frac{C}{\varepsilon^{2}}, \quad \frac{g}{S_{t}}=\frac{h k_{m e}}{S+\frac{\delta h^{2}}{2} k_{m m}}, \quad \eta=\frac{l_{p}}{\varepsilon} .
\end{aligned}
$$

The physical meanings of the parameters of the piezo-composite plate above introduced are $\rho_{t}$, effective mass per unit surface; $S_{t}$, effective bending stiffness; $v_{t}$, effective Poisson ratio; $c$, effective capacitance per unit surface; $g$, effective piezoelectric coupling; $\eta$, piezoelectric covering factor.

On the other hand, the total magnetic energy of the homogenized electric (6) becomes 


$$
\begin{aligned}
U_{\text {hom }}^{e}= & \frac{1}{2} S_{e} \int_{\Omega}\left(\left(\partial_{11} \psi\right)^{2}+\left(\partial_{22} \psi\right)^{2}+2 v_{t}\left(\partial_{11} \psi\right)\left(\partial_{22} \psi\right)\right. \\
& \left.+2\left(1-v_{t}\right)\left(\partial_{12} \psi\right)^{2}\right) \mathrm{d} \Omega,
\end{aligned}
$$

where in order to guarantee the multiresonance condition, the electric parameters are chosen according to (7) upon substituting $S$ with $S_{t}, v$ with $v_{t}, \rho h$ with $\rho_{t}$ and introducing

$S_{e}=\frac{L_{1}+L_{2}}{n^{4} L_{1} L_{2}} \varepsilon^{2}$.

The total Lagrangian of the resulting homogenized PEM plate is given by $\mathscr{L}_{\text {hom }}^{\text {PEM }}=\mathscr{L}_{\text {hom }}^{p-c}-U_{\text {hom }}^{e}$.

The equations of motion, together with the admissible boundary conditions are obtained by setting to zero the first variation of the total action between two prescribed time instants $t_{i}$ and $t_{f}$, say $\mathscr{A}_{\text {hom }}$, on the set of isochronous motions:

$\delta \mathscr{A}_{\text {hom }}=0$,

where

$\mathscr{A}_{\text {hom }}=\int_{t_{i}}^{t_{f}} \mathscr{L}_{\mathrm{hom}}^{\mathrm{PEM}} \mathrm{d} t$.

By computing the first variation of $\mathscr{A}$ and after several applications of Green's theorem we get ${ }^{4}$

$$
\begin{aligned}
\delta \mathscr{A}_{\text {hom }}= & -\int_{t_{i}}^{t_{f}} \int_{\Omega}\left[\left(S_{t} \nabla^{4} u+\rho_{t} \ddot{u}+g \nabla^{2} \dot{\psi}\right) \delta u\right. \\
& \left.+\left(S_{e} \nabla^{4} \psi+c \ddot{\psi}-g \nabla^{2} \dot{u}\right) \delta \psi\right] \mathrm{d} \Omega \mathrm{d} t \\
& +\int_{t_{i}}^{t_{f}} \int_{x_{1}}\left(\left[S_{t}\left(\partial_{22} u+v_{t} \partial_{11} u\right)-g \dot{\psi}\right] \partial_{2} \delta u\right. \\
& \left.+\left[-S_{t}\left(\partial_{222} u+\left(2-v_{t}\right) \partial_{112} u\right)+g \partial_{2} \dot{\psi}\right] \delta u\right) \mathrm{d} x_{1} \mathrm{~d} t \\
& +\int_{t_{i}}^{t_{f}} \int_{x_{1}}\left(\left[S_{t}\left(\partial_{22} \psi+v_{t} \partial_{11} \psi\right)\right] \partial_{2} \delta \psi\right. \\
& \left.+\left[-S_{t}\left(\partial_{222} \psi+\left(2-v_{t}\right) \partial_{112} \psi\right)\right] \delta \psi\right) \mathrm{d} x_{1} \mathrm{~d} t \\
& +\int_{t_{i}}^{t_{f}} \int_{x_{2}}\left(-\left[S_{t}\left(\partial_{11} u+v_{t} \partial_{22} u\right)-g \dot{\psi}\right] \partial_{1} \delta u\right. \\
& \left.-\left[-S_{t}\left(\partial_{111} u+\left(2-v_{t}\right) \partial_{122} u\right)+g \partial_{1} \dot{\psi}\right] \delta u\right) \mathrm{d} x_{2} \mathrm{~d} t \\
& +\int_{t_{i}}^{t_{f}} \int_{x_{2}}\left(-\left[S_{t}\left(\partial_{11} \psi+v_{t} \partial_{22} \psi\right)\right] \partial_{1} \delta \psi\right. \\
& \left.-\left[-S_{t}\left(\partial_{111} \psi+\left(2-v_{t}\right) \partial_{122} \psi\right)\right] \delta \psi\right) \mathrm{d} x_{2} \mathrm{~d} t .
\end{aligned}
$$

By setting to zero the first variation of $\mathscr{A}_{\text {hom }}$, we obtain the following evolution equations:

$$
\left\{\begin{array}{l}
S_{t} \nabla^{4} u+\rho_{t} \ddot{u}+g \nabla^{2} \dot{\psi}=0, \\
S_{e} \nabla^{4} \psi+c \ddot{\psi}-g \nabla^{2} \dot{u}=0,
\end{array}\right.
$$

together with the following homogeneous boundary conditions:

at an $x_{1}=$ constant edge:

$$
\left\{\begin{array}{l}
\partial_{11} \psi+v_{t} \partial_{22} \psi=0 \text { or } \partial_{1} \psi=0 \\
\partial_{111} \psi+\left(2-v_{t}\right) \partial_{122} \psi=0 \text { or } \psi=0 \\
S_{t}\left(\partial_{11} u+v_{t} \partial_{22} u\right)-g \dot{\psi}=0 \text { or } \partial_{1} u=0 \\
S_{t}\left(\partial_{111} u+\left(2-v_{t}\right) \partial_{122} u\right)-g \partial_{1} \dot{\psi}=0 \text { or } u=0
\end{array}\right.
$$

at an $x_{2}=$ constant edge:

$$
\left\{\begin{array}{l}
\partial_{22} \psi+v_{t} \partial_{11} \psi=0 \text { or } \partial_{2} \psi=0 \\
\partial_{222} \psi+\left(2-v_{t}\right) \partial_{112} \psi=0 \text { or } \psi=0 \\
S_{t}\left(\partial_{22} u+v_{t} \partial_{11} u\right)-g \dot{\psi}=0 \text { or } \partial_{2} u=0 \\
S_{t}\left(\partial_{22} u+\left(2-v_{t}\right) \partial_{112} u\right)-g \partial_{2} \dot{\psi}=0 \text { or } u=0 .
\end{array}\right.
$$

For future developments, it is convenient to nondimensionalize Eqs. (11) by introducing $u_{0}, \psi_{0}, t_{0}$ and $l_{0}$ defined as characteristic deflection, flux-linkage, time and length, respectively. Therefore, Eqs. (11) become ${ }^{5}$

$$
\left\{\begin{array}{l}
\nabla^{4} u+\alpha^{4} \ddot{u}-\beta^{2} \nabla^{2} \dot{\psi}=0, \\
\nabla^{4} \psi+\alpha^{4} \ddot{\psi}+\beta^{2} \nabla^{2} \dot{u}=0,
\end{array}\right.
$$

\footnotetext{
${ }^{5}$ Since it does not cause misunderstandings, the same notation will be adopted also for the dimensionless governing equations.
} 
where

$\alpha^{4}=\frac{\rho_{t}}{S_{t}} \frac{l_{0}^{4}}{t_{0}^{2}}, \quad \beta^{2}=-g \frac{1}{S_{t}} \sqrt{\frac{\rho_{t}}{c}} \frac{l_{0}^{2}}{t_{0}}$,

and $u_{0}$ and $\psi_{0}$ have been chosen according to

$\frac{\psi_{0}}{u_{0}}=\sqrt{\frac{S_{t}}{S_{e}}} \equiv \sqrt{\frac{\rho_{t}}{c}}$,

in order to preserve the gyroscopic coupling.

\section{Simply-supported PEM plates}

\subsection{Free undamped vibrations}

In the present subsection, the free vibrations of simply supported PEM plates will be studied. By a simply supported PEM plate, we mean a simply supported piezo-composite plate controlled by the aforementioned analog circuit subjected, itself, to simply supporting electrical boundary conditions. The electromechanical boundary conditions are

at an $x_{1}=$ constant edge: $\left\{\begin{array}{l}\partial_{11} \psi=0, \\ \psi=0, \\ \partial_{11} u=0, \\ u=0,\end{array}\right.$

at an $x_{2}=$ constant edge: $\left\{\begin{array}{l}\partial_{22} \psi=0, \\ \psi=0, \\ \partial_{22} u=0, \\ u=0,\end{array}\right.$

which are admissible in the sense of (12). Let us underline, that there are not mixed electromechanical boundary conditions. The electrical boundary conditions are chosen to mimic the mechanical ones, prescribed by the external fixed constraints, as suggested by [26].

The electromechanical fields $(u, \psi)$ can be expressed as follows:

$$
\left\{\begin{array}{l}
u\left(x_{1}, x_{2}, t\right)=\sum_{h, k} p_{h k}(t) m_{h k}\left(x_{1}, x_{2}\right), \\
\psi\left(x_{1}, x_{2}, t\right)=\sum_{h, k} q_{h k}(t) m_{h k}\left(x_{1}, x_{2}\right),
\end{array}\right.
$$

where $p_{h k}(t)$ and $q_{h k}(t)$ are the time depending Fourier coefficients of the normalized eigenfunctions $m_{h k}(x, y)$ coming from the eigenvalue problem

$$
\nabla^{4} m_{h k}=\lambda_{h k}^{4} m_{h k} \text {, }
$$

with the simply supporting boundary conditions:

$$
\begin{array}{ll}
\text { at an } x_{1}=\text { constant edge: } & \left\{\begin{array}{l}
\partial_{11} m=0, \\
m=0,
\end{array}\right. \\
\text { at an } x_{2}=\text { constant edge: }\left\{\begin{array}{l}
\partial_{22} m=0, \\
m=0 .
\end{array}\right.
\end{array}
$$

The computation of the modal characteristics $m_{h k}$ and $\lambda_{h k}$ leads to

$m_{h k}\left(x_{1}, x_{2}\right)=2 \sin \left(h \pi x_{1}\right) \sin \left(k \pi x_{2}\right), \quad \lambda_{h k}^{4}=\pi^{4}\left[h^{2}+k^{2}\right]^{2}$,

where the characteristic length $l_{0}$ has been chosen equal to the plate edge $l$. Let us mention the following properties of $m_{h k}$ : they are orthonormal, i.e.,

$\int_{\Omega} m_{h k} m_{n l} \mathrm{~d} \Omega=1 \quad$ if $(h, k)=(n, l)$ and 0 otherwise

and they are simultaneously eigenfunctions of the Laplacian operator with Dirichlet boundary conditions, i.e.,

$\nabla^{2} m_{h k}=-\lambda_{h k}^{2} m_{h k}$.

Introducing the previous expansions (14) into (13) and projecting onto the given basis, it is easy to derive the following infinite set of ODEs governing the electrical and mechanical Fourier coefficients $p_{h k}(t), q_{h k}(t)$

$\left\{\begin{array}{l}\lambda_{h k}^{4} p_{h k}+\alpha^{4} \ddot{p}_{h k}+\beta^{2} \lambda_{h k}^{2} \dot{q}_{h k}=0, \\ \lambda_{h k}^{4} q_{h k}+\alpha^{4} \ddot{q}_{h k}-\beta^{2} \lambda_{h k}^{2} \dot{p}_{h k}=0,\end{array} \quad(h, k) \in \mathbb{N}^{2}\right.$.

We underline that in this instance of PEM plate, the $(h, k)$ mechanical mode remains uncoupled to other modes after the introduction of the control network. Therefore, no spillover phenomena occur. The electrically simply supported edges can be synthesized by connecting to ground the corresponding nodes of the boundary modules.

Each of the coupled ODEs in (15), can be conveniently written in the first-order state-space form:

$$
\begin{aligned}
& \dot{\mathbf{x}}_{h k}(t)=\mathbf{A}_{h k} \mathbf{x}_{h k}(t), \\
& \mathbf{x}_{h k}(0)=\mathbf{x}_{h k}^{0},
\end{aligned}
$$

where the vector variable $\mathbf{x}_{h k}$, the state space matrix $\mathbf{A}_{h k}$ and the initial conditions $\mathbf{x}_{h k}^{0}$ are

$$
\begin{aligned}
\mathbf{x}_{h k} & =\left\{\begin{array}{l}
p_{h k} \\
q_{h k} \\
\dot{p}_{h k} \\
\dot{q}_{h k}
\end{array}\right\}, \quad \mathbf{A}_{h k}=\left[\begin{array}{cccc}
0 & 0 & 1 & 0 \\
0 & 0 & 0 & 1 \\
-\frac{\lambda_{h k}^{4}}{\alpha^{4}} & 0 & 0 & -\frac{\beta^{2}}{\alpha^{4}} \lambda_{h k}^{2} \\
0 & -\frac{\lambda_{h k}^{4}}{\alpha^{4}} & \frac{\beta^{2}}{\alpha^{4}} \lambda_{h k}^{2} & 0
\end{array}\right], \\
\mathbf{x}_{h k}^{0} & =\left\{\begin{array}{l}
p_{h k}^{0} \\
q_{h k}^{0} \\
\dot{p}_{h k}^{0} \\
\dot{q}_{h k}^{0}
\end{array}\right\} .
\end{aligned}
$$

The solution of (17) can be expressed in the following form:

$\mathbf{x}_{h k}(t)=\mathrm{e}^{\mathbf{A}_{h k} t} \mathbf{x}_{h k}^{0}=\mathbf{V}_{h k} \mathrm{e}^{\mathbf{V}_{h k}^{-1} \mathbf{A}_{h k} \mathbf{V}_{h k} t}\left(\mathbf{V}_{h k}^{-1} \mathbf{x}_{h k}^{0}\right)$,

where the columns of the matrix $\mathbf{V}_{h k}$ are the eigenvectors of the matrix $\mathbf{A}_{h k}$. The set $\boldsymbol{\Omega}_{h k}$ of eigenvalues (ordered as a four-dimensional complex vector) of $\mathbf{A}_{h k}$ has the following simple form: 

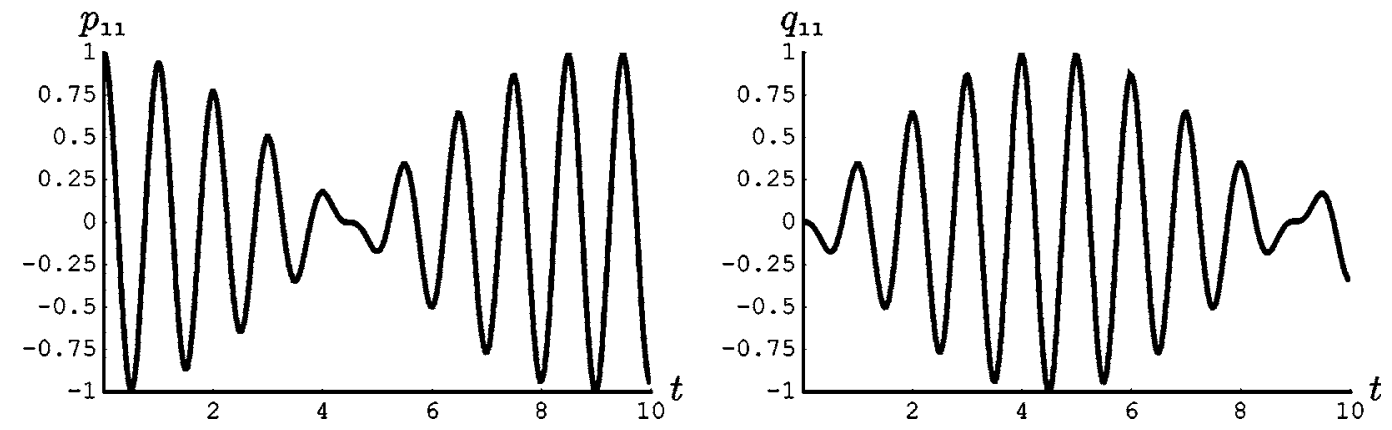

Fig. 5. Time evolution of $p_{11}$ and $q_{11}$ for an initial unitary mechanical deflection on mode $(1,1)$.

$\boldsymbol{\Omega}_{h k}=\mathrm{I}\left\{\begin{array}{c}\omega_{h k}^{c}-\omega_{h k}^{m} \\ -\left(\omega_{h k}^{c}-\omega_{h k}^{m}\right) \\ \omega_{h k}^{c}+\omega_{h k}^{m} \\ -\left(\omega_{h k}^{c}+\omega_{h k}^{m}\right)\end{array}\right\}$

in which "I" is the imaginary unit, and the so-called carrier and the modulating frequencies $\omega_{h k}^{c}$ and $\omega_{h k}^{m}$ defined by

$\omega_{h k}^{c}=\frac{\lambda_{h k}^{2}}{\alpha^{4}} \frac{1}{2} \sqrt{\beta^{4}+4 \alpha^{4}}, \quad \omega_{h k}^{m}=\frac{1}{2} \lambda_{h k}^{2} \frac{\beta^{2}}{\alpha^{4}}$,

have been introduced. We explicitly remark that the eigenvalues in $\boldsymbol{\Omega}_{h k}$ consist of two pairs of purely imaginary complex conjugate numbers because of the assumed absence of dissipative phenomena. From (19) it may be noted that the ratio $\frac{\omega_{h k}^{m}}{\omega_{\omega_{k}}^{k}}$ is completely mode-independent, i.e.,

$\frac{\omega_{h k}^{m}}{\omega_{h k}^{c}}=\frac{1}{\sqrt{1+4 \frac{\alpha^{4}}{\beta^{4}}}}$.

Moreover, the eigenvectors matrix $\mathbf{V}_{h k}$ and its inverse $\mathbf{V}_{h k}^{-1}$ are given by

$\mathbf{V}_{h k}=\left[\begin{array}{cccc}\mathrm{I} & -\mathrm{I} & \mathrm{I} & -\mathrm{I} \\ 1 & 1 & 1 & 1 \\ -\omega_{h k}^{m}-\omega_{h k}^{c} & -\omega_{h k}^{m}-\omega_{h k}^{c} & \omega_{h k}^{c}-\omega_{h k}^{m} & \omega_{h k}^{c}-\omega_{h k}^{m} \\ \mathrm{I}\left(\omega_{h k}^{c}+\omega_{h k}^{m}\right) & -\mathrm{I}\left(\omega_{h k}^{c}+\omega_{h k}^{m}\right) & \mathrm{I}\left(-\omega_{h k}^{c}+\omega_{h k}^{m}\right) & -\mathrm{I}\left(-\omega_{h k}^{c}+\omega_{h k}^{m}\right)\end{array}\right]$,

$\mathbf{V}_{h k}^{-1}=\frac{1}{4 \omega_{h k}^{c}}\left[\begin{array}{cccc}\mathrm{I}\left(-\omega_{h k}^{c}+\omega_{h k}^{m}\right) & \omega_{h k}^{c}-\omega_{h k}^{m} & -1 & -\mathrm{I} \\ -\mathrm{I}\left(-\omega_{h k}^{c}+\omega_{h k}^{m}\right) & \omega_{h k}^{c}-\omega_{h k}^{m} & -1 & \mathrm{I} \\ -\mathrm{I}\left(\omega_{h k}^{c}+\omega_{h k}^{m}\right) & \omega_{h k}^{c}+\omega_{h k}^{m} & 1 & \mathrm{I} \\ \mathrm{I}\left(\omega_{h k}^{c}+\omega_{h k}^{m}\right) & \omega_{h k}^{c}+\omega_{h k}^{m} & 1 & -\mathrm{I}\end{array}\right]$.

Let us explicitly derive the solutions of (16) for two significative initial conditions.

- Initial purely mechanical deformation of the PEM plate on its $(h, k)$-mode:

$u\left(x_{1}, x_{2}, 0\right)=p_{0} m_{h k}\left(x_{1}, x_{2}\right)$, thus

$\mathbf{x}_{h k}^{0}=\left\{\begin{array}{c}p_{0} \\ 0 \\ 0 \\ 0\end{array}\right\} \quad$ and $\quad \mathbf{x}_{i j}^{0}=\left\{\begin{array}{l}0 \\ 0 \\ 0 \\ 0\end{array}\right\} \forall(i, j) \neq(h, k)$.

Consequently the general solution (18) becomes

$u\left(x_{1}, x_{2}, t\right)$

$=p_{0}\left(\cos \omega_{h k}^{c} t \cos \omega_{h k}^{m} t+\frac{\omega_{h k}^{m}}{\omega_{h k}^{c}} \sin \omega_{h k}^{c} t \sin \omega_{h k}^{m} t\right) m_{h k}\left(x_{1}, x_{2}\right)$.

For the sake of completeness we report the unique non-vanishing Fourier coefficients vector $\mathbf{x}_{h k}$ :

$\mathbf{x}_{h k}=\left\{\begin{array}{c}p_{0}\left(\cos \omega_{h k}^{c} t \cos \omega_{h k}^{m} t+\frac{\omega_{h k}^{m}}{\omega_{h k}^{m}} \sin \omega_{h k}^{c} t \sin \omega_{h k}^{m} t\right) \\ p_{0}\left(\cos \omega_{h k}^{c} t \sin \omega_{h k}^{m} t-\frac{\omega_{h k}^{m}}{\omega_{h k}^{m}} \sin \omega_{h k}^{c} t \cos \omega_{h k}^{m} t\right) \\ p_{0} \frac{\left(\omega_{h k}^{m}\right)^{2}-\left(\omega_{h k}^{c}\right)^{2}}{\omega_{h k}^{c}} \sin \omega_{h k}^{c} t \cos \omega_{h k}^{m} t \\ p_{0} \frac{-\left(\omega_{h k}^{c}\right)^{2}+\left(\omega_{h k}^{m}\right)^{2}}{\omega_{h k}^{c}} \sin \omega_{h k}^{c} t \sin \omega_{h k}^{m} t\end{array}\right\}$.

In Fig. 5 the evolutions of $p_{11}(t)$ and $q_{11}(t)$ for an initial deformation on mode $(1,1)$ for the considered aluminum PEM plate described in Appendix A. Let us remark that the evolution in Fig. 5 shows a beating/amplitude modulation phenomenon between the electrical and mechanical subsystems: by using the language of the theory of signals we can say that the effect of the piezoelectric distributed coupling together with the analog circuit introduces an amplitude modulation of the mechanical signal, in which the carrier frequency $\omega_{h k}^{c}$ is close (when $\beta$ is small) to that of the uncoupled mechanical mode and the modulating frequency $\omega_{h k}^{m}$ is proportional to the coupling effectiveness (see definitions (19)).

- Point impact at the plate center. This correspond to assign an initial velocity $v_{0}$ to the central point of the plate surface: ${ }^{6}$

\footnotetext{
${ }^{6}$ The symbol $\delta$ represent the Dirac delta generalized function.
} 
$\dot{u}\left(x_{1}, x_{2}, 0\right)=v_{0} \delta\left(x_{1}-\frac{1}{2}\right) \delta\left(x_{2}-\frac{1}{2}\right)=\sum_{h, k} \dot{p}_{h k}^{0} m_{h k}\left(x_{1}, x_{2}\right)$

hence the initial conditions for the modal evolution become

$\mathbf{x}_{h k}^{0}=\left\{\begin{array}{c}0 \\ 0 \\ \dot{p}_{h k}^{0} \\ 0\end{array}\right\}$,

where the Fourier coefficients $\dot{p}_{h k}^{0}$ are

$$
\begin{aligned}
\dot{p}_{h k}^{0} & =v_{0} \int_{\Omega} \delta\left(x_{1}-\frac{1}{2}\right) \delta\left(x_{2}-\frac{1}{2}\right) m_{h k}\left(x_{1}, x_{2}\right) \mathrm{d} \Omega \\
& =v_{0} m_{h k}\left(\frac{1}{2}, \frac{1}{2}\right)=v_{0} 2 \sin \left(\frac{h \pi}{2}\right) \sin \left(\frac{k \pi}{2}\right) \\
& =v_{0} 2\left[(-1)^{(h-1) / 2} \frac{1-(-1)^{h}}{2}\right]\left[(-1)^{(k-1) / 2} \frac{1-(-1)^{k}}{2}\right]
\end{aligned}
$$

and the solution (18) becomes

$u\left(x_{1}, x_{2}, t\right)=\sum_{h, k}\left(\frac{\dot{p}_{h k}^{0}}{\omega_{h k}^{c}} \sin \omega_{h k}^{c} t \cos \omega_{h k}^{m} t\right) m_{h k}\left(x_{1}, x_{2}\right)$.

For the sake of completeness we report the generic Fourier coefficients vector $\mathbf{x}_{h k}$ :

$$
\mathbf{x}_{h k}=\left\{\begin{array}{c}
\frac{\dot{p}_{h k}^{0}}{\omega_{h k}^{c}} \sin \omega_{h k}^{c} t \cos \omega_{h k}^{m} t \\
\dot{p}_{h k}^{0} \sin \omega_{h k}^{c} t \sin \omega_{h k}^{m} t \\
\dot{p}_{h k}^{0}\left(\cos \omega_{h k}^{c} t \cos \omega_{h k}^{m} t-\frac{\omega_{m}}{\omega_{h k}^{c}} \sin \omega_{h k}^{c} t \sin \omega_{h k}^{m} t\right) \\
\dot{p}_{h k}^{0}\left(\cos \omega_{h k}^{c} t \sin \omega_{h k}^{m} t+\frac{\omega_{m}}{\omega_{h k}^{c}} \sin \omega_{h k}^{c} t \cos \omega_{h k}^{m} t\right)
\end{array}\right\} .
$$

\subsection{Optimal attenuation in forced vibrations}

The results of the analysis of non-dissipative PEM plates, presented in the previous subsection, assess the rapidity of the energy transfer from the mechanical to the electrical form (and vice versa). These high performances are mainly due to the internal resonance established in between the mechanical and the electrical subsystems.

In this section, we will consider the effect of electrical dissipation on mechanical forced oscillations, and establish optimal resistances to be inserted in the analog circuit. Towards this goal, we introduce an external mechanical disturbance, say $f\left(x_{1}, x_{2}, t\right)$, and an electrical dissipation in (13) as follows:

$$
\left\{\begin{array}{l}
\nabla^{4} u+\alpha^{4} \ddot{u}-\beta^{2} \nabla^{2} \dot{\psi}=f \\
\nabla^{4} \psi+\alpha^{4} \ddot{\psi}+\beta^{2} \nabla^{2} \dot{u}+\mathbf{D}[\dot{\psi}]=0
\end{array}\right.
$$

where D indicates an arbitrary self-adjoint, positive semi-definite operator sharing the same eigenfunctions of $\nabla^{4}$. The assumptions on the nature of the electric dissipation lead to the following advantages:

- proportional damping: the modal shapes of the dissipative PEM plate are the same of those of the nondissipative one;

- a simple realization of the dissipative circuit using only resistors is possible.

Consequently, we introduce the modal forcing term $f_{h k}$ in (16) and introduce the modal electric damping $\gamma_{h k}$ in the state matrix $\mathbf{A}_{h k}$ of (17):

$$
\begin{aligned}
& \dot{\mathbf{x}}_{h k}(t)=\mathbf{A}_{h k} \mathbf{x}_{h k}(t)+\left\{\begin{array}{llll}
0 & 1 & 0 & 0
\end{array}\right\}^{T} f_{h k}, \\
& \mathbf{A}_{h k}=\left[\begin{array}{cccc}
0 & 0 & 1 & 0 \\
0 & 0 & 0 & 1 \\
-\frac{\lambda_{h k}^{4}}{\alpha^{4}} & 0 & 0 & -\frac{\beta^{2}}{\alpha^{4}} \lambda_{h k}^{2} \\
0 & -\frac{\lambda_{h k}^{4}}{\alpha^{4}} & \frac{\beta^{2}}{\alpha^{4}} \lambda_{h k}^{2} & -\frac{\gamma_{h k}}{\alpha^{4}}
\end{array}\right] .
\end{aligned}
$$

Next, we introduce the modal mobility $H_{h k}(\omega)$ defined as the ratio of the Fourier transform (denoted by $\mathscr{F})$ of the modal mechanical velocity to the Fourier transform of the modal force, i.e.,

$$
\begin{aligned}
& H_{h k}(\omega) \\
& =\frac{\mathscr{F}\left[\dot{p}_{h k}\right]}{\mathscr{F}\left[f_{h k}\right]} \\
& =\frac{\alpha^{4} \mathrm{I} \omega\left(-\alpha^{4} \omega^{2}+\lambda_{h k}^{4}+\mathrm{I} \gamma_{h k} \omega\right)}{\alpha^{8} \omega^{4}-\mathrm{I} \alpha^{4} \gamma_{h k} \omega^{3}+\left(-2 \alpha^{4} \lambda_{h k}^{4}-\beta^{4} \lambda_{h k}^{4}\right) \omega^{2}+\mathrm{I} \gamma_{h k} \lambda_{h k}^{4} \omega+\lambda_{h k}^{8}},
\end{aligned}
$$

where $\omega$ indicates the dimensionless angular frequency.

The optimization problem we want to solve is to find $\gamma_{h k}$ minimizing the maximum value ${ }^{7}$ of $\left|H_{h k}\right|$. Once the optimal expression of the modal electric dampings $\gamma_{h k}$ are known, we can construct the optimal dissipation operator $\mathbf{D}[\dot{\psi}]$ from its spectral representation

$$
\mathbf{D}[\dot{\psi}]=\sum_{h, k} \gamma_{h k}\left[\int_{\Omega} \dot{\psi}\left(x_{1}, x_{2}, t\right) m_{h k}\left(x_{1}, x_{2}\right) \mathrm{d} \Omega\right] m_{h k}\left(x_{1}, x_{2}\right) \text {. }
$$

Following [8], it can be shown that there exists two points: $S_{h k}=\left(\omega_{h k}^{\mathrm{S}},\left|H_{h k}\left(\omega_{h k}^{\mathrm{S}}\right)\right|\right)$ and $T_{h k}=\left(\omega_{h k}^{\mathrm{T}},\left|H_{h k}\left(\omega_{h k}^{\mathrm{T}}\right)\right|\right)$ (called fixed points) independent of the damping parameter $\gamma_{h k}$. These two fixed points may be found be equating $\left|H_{h k}\right|^{2}$ evaluated when $\gamma_{h k} \rightarrow \infty$ (absence of the

\footnotetext{
${ }^{7}$ This optimality criterion is often called $\mathscr{H}_{\infty}$ optimization (see, e.g., [28]).
} 
analog circuit) with $\left|H_{h k}\right|^{2}$ evaluated when $\gamma_{h k}=0$ (nondissipative PEM), namely,

$\omega_{h k}^{\mathrm{T}, \mathrm{S}}=\frac{\lambda_{h k}^{2}}{2 \alpha^{4}} \sqrt{\left(4 \alpha^{4}+\beta^{4}\right) \pm \beta^{2} \sqrt{8 \alpha^{4}+\beta^{4}}}$,

$\left|H_{h k}\left(\omega_{h k}^{\mathrm{S}}\right)\right| \equiv\left|H_{h k}\left(\omega_{h k}^{\mathrm{T}}\right)\right|=\frac{\sqrt{2} \alpha^{4}}{\beta^{2} \lambda_{h k}^{2}}$.

In order to minimize the maximum of $\left|H_{h k}\right|$, we compel $\left|H_{h k}\right|$ to attain its maxima at the fixed point frequencies $\omega_{h k}^{\mathrm{T}, \mathrm{S}}$, i.e.,

$\left.\frac{\mathrm{d}}{\mathrm{d} \omega}\left|H_{h k}\right|\right|_{\omega=\omega_{h k}^{\mathrm{S}}}=0,\left.\quad \frac{\mathrm{d}}{\mathrm{d} \omega}\left|H_{h k}\right|\right|_{\omega=\omega_{h k}^{\mathrm{T}}}=0$,

which yields

$\gamma_{h k}=\sqrt{\frac{3}{2}} \beta^{2} \lambda_{h k}^{2}, \quad \max _{\omega \in \mathbb{R}}\left|H_{h k}(\omega)\right|=\frac{\sqrt{2} \alpha^{4}}{\beta^{2} \lambda_{h k}^{2}}$.

From the representation (21) and the optimal estimation for the damping parameters in (22), the optimal electrical dissipative operator becomes

$\mathbf{D}[\dot{\psi}]=-\sqrt{\frac{3}{2}} \beta^{2} \nabla^{2} \dot{\psi}$.

In Fig. 6 we show the frequency responses $\left|H_{11}\right|$ of the considered PEM plate for different electric dampings $\gamma_{11}$ : optimal (solid), vanishing (dash-dotted), infinity (dashed).

Using a synthesis procedure similar to the one used to obtain the analog circuit in Fig. 1 we get the circuit sketched in Fig. 7, where from (23) the optimal resistance $R$ is given by

$R=-\frac{1}{g} \sqrt{\frac{2}{3}} \sqrt{\frac{\rho_{t}}{c}}$.

Let us remark that the found dissipative network assures the optimal suppression of forced oscillation for any mechanical mode.

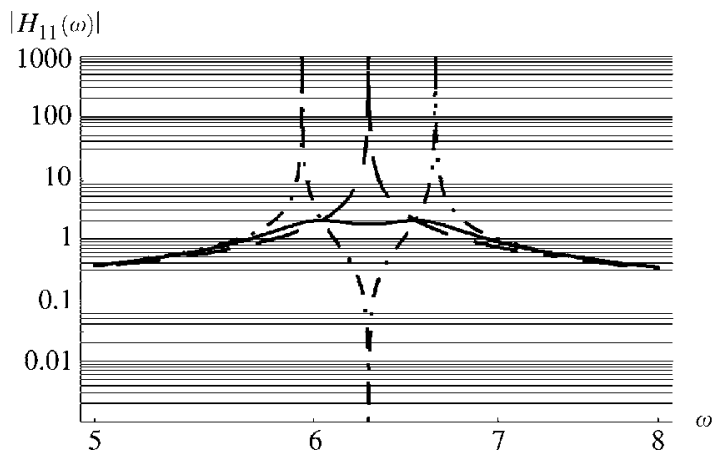

Fig. 6. Absolute value of the modal mobility function for mode $(1,1)$.

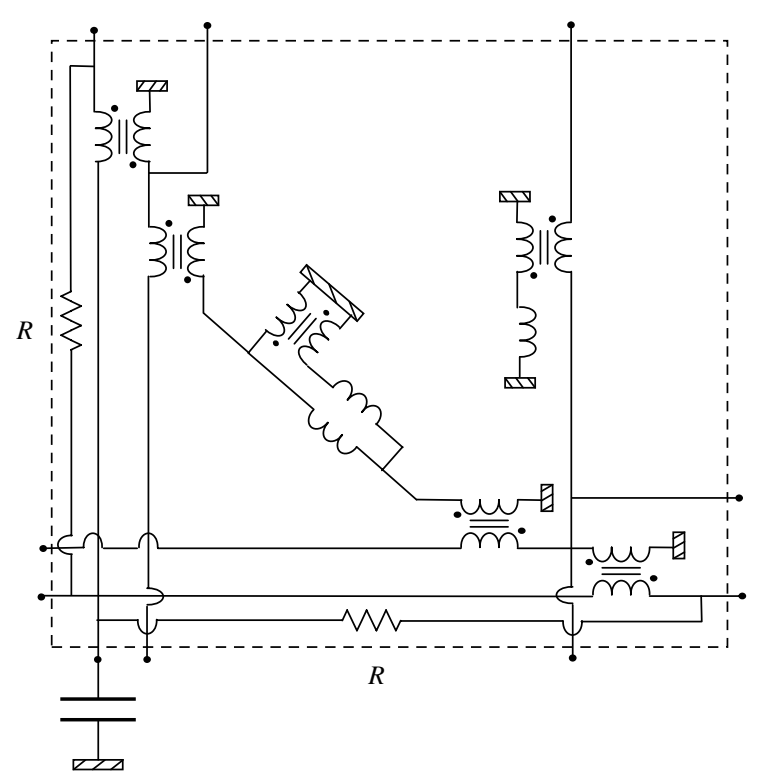

Fig. 7. Sketch of the analog circuit endowed with optimal dissipation.

\section{Conclusions}

In this paper a novel passive technique for damping multimodal vibrations of thin plates has been developed. The proposed methodology is based on the concept of Piezo-ElectroMechanical structures (see, e.g., [7]), i.e., coupling the host structure to its electric analog, exploiting distributed piezoelectric transduction. The analog circuit may be regarded as a dynamic controller which resonates at the same frequencies as the host structure and, in addition, responds by driving every transducer with the electric potential needed to suppress the excited mechanical modal shapes.

The problem of finding a completely passive realization of the analog circuit of a Kirchhoff-Love plate was, to our knowledge, unsolved (see, e.g., [1]) before the present work. The derivation of the model of the PEM plate was done by constructing a global Lagrangian for the resulting electromechanical system constituted by the piezo-composite plate and the analog network. In this way it was possible to rigorously derive the set of admissible boundary conditions for both the mechanical and electrical variables. The free vibrations of a nondissipative simply supported PEM plate under different initial conditions were studied and analytical results were exhibited. Finally, the problem of determining optimal resistors to be inserted in the analog circuit for broadband suppression of mechanical disturbances was solved.

All the numerical simulations were performed on an aluminum simply supported plate. The presented results 
show the practical effectiveness and technical feasibility of the proposed device in the multimodal suppression of mechanical disturbances.

Further developments of this work will include the analysis of PEM plate subjected to more complicated boundary conditions and the developments of PEM thin shells. Even if the fundamental equations of arbitrarily constrained PEM plate were derived in this paper, we believe that great efforts will be needed when studying free oscillations and optimizing resistive networks for not simply-supported edges. Indeed, in the general case mixed electromechanical boundary conditions arise and the optimal dissipative operator may not be a differential one.

\section{Acknowledgments}

The partial support of the Engineering Science and Mechanics Department of the Virginia Polytechnic Institute and State University is gratefully acknowledged by the authors. This presented research has been also partially supported by MIUR, Ministero per l'Innovazione, l'Università e la Ricerca Fondi Ricerca PRIN "Metodi innovativi per l'analisi e il controllo di sistemi vibroacustici" (protocollo 200309593_2004) and by the Università di Roma "La Sapienza" Progetto di Ateneo "Soppressione delle vibrazioni mediante modifiche strutturali: modelli continui e discreti" (codice progetto C26A038198). The authors would like to thank the reviewers for their comments that led to a considerable improvement in the manuscript.

\section{Appendix A}

All the numerical results shown on the plots presented in the previous sections have been realized considering a square plate made of aluminum, whose geometry is described in Table 1 . The plate is assumed to be covered, on both the faces, by $5 \times 5$ piezoceramic transducers (number T110-H4E-602 Piezo Systems, Inc.) having the properties listed in Table 2. The corresponding values for the analog circuital elements are computed in Table 3.

The characteristics quantities used in the non-dimensionalization are reported in Table 4 (the characteristic

Table 1

Properties and dimensions of the host plate

\begin{tabular}{lll}
\hline Coefficient & Value & Units \\
\hline Edge $(l)$ & 1 & $\mathrm{~m}$ \\
Thickness $(h)$ & $2 \times 10^{-3}$ & $\mathrm{~m}$ \\
Young's modulus $(E)$ & $70 \times 10^{9}$ & $\mathrm{~Pa}$ \\
Poisson ratio $(v)$ & 0.3 & \\
Mass density $(\rho)$ & 2700 & $\mathrm{~kg} \mathrm{~m}^{-3}$ \\
\hline
\end{tabular}

Table 2

Properties and dimensions of the piezoelectric sheets

\begin{tabular}{lll}
\hline Coefficient & Value & Units \\
\hline Young's modulus $\left(E_{p}\right)$ & $62 \times 10^{9}$ & $\mathrm{~Pa}$ \\
Poisson ratio $\left(v_{p}\right)$ & 0.31 & \\
Piezoelectric constant $\left(d_{31}\right)$ & $-320 \times 10^{-12}$ & $\mathrm{~m} \mathrm{~V}^{-1}$ \\
Dielectric constant $\left(e_{33}^{\mathrm{T}}\right)$ & $33.63 \times 10^{-9}$ & $\mathrm{~F} \mathrm{~m}^{-1}$ \\
Thickness $(\delta)$ & $0.267 \times 10^{-3}$ & $\mathrm{~m}$ \\
Mass density $\left(\rho_{p}\right)$ & 7800 & $\mathrm{~kg} \mathrm{~m}^{-3}$ \\
Edge $\left(l_{p}\right)$ & $5 \times 10^{-2}$ & $\mathrm{~m}$ \\
Coverage factor $(\eta)$ & $25 \%$ & \\
Sampling step $(\varepsilon)$ & .2 & $\mathrm{~m}$ \\
\hline
\end{tabular}

Table 3

Nominal values of the circuital elements

\begin{tabular}{lll}
\hline Coefficient & Value & Units \\
\hline "First" inductance $\left(n^{4} L_{1}\right)$ & 1723 & $\mathrm{H}$ \\
"Second" inductance $\left(n^{4} L_{2}\right)$ & 928 & $\mathrm{H}$ \\
"Third" inductance $\left(n^{4} L_{3}\right)$ & 431 & $\mathrm{H}$ \\
Piezoelectric capacitance $(C)$ & $285.1 \times 10^{-9}$ & $\mathrm{~F}$ \\
Resistance $(R)$ & $338.0 \times 10^{3}$ & $\Omega$ \\
\hline
\end{tabular}

Table 4

Characteristic quantities used in the non-dimensionalization

\begin{tabular}{lll}
\hline Coefficient & Value & Units \\
\hline Characteristic length $\left(l_{0}\right)$ & 1 & $\mathrm{~m}$ \\
Characteristic deflection $\left(u_{0}\right)$ & $10^{-3}$ & $\mathrm{~m}$ \\
Characteristic time $\left(t_{0}\right)$ & 0.104 & $\mathrm{~s}$ \\
Characteristic flux-linkage $\left(\psi_{0}\right)$ & 0.891 & $\mathrm{~V} \mathrm{~s}$ \\
\hline
\end{tabular}

time has been chosen equal to the period of oscillation of the first mode of the simply supported piezo-composite plate).

\section{References}

[1] Alessandroni S, dell'Isola F, Porfiri M. A revival of electric analogs for vibrating mechanical systems aimed to their efficient control by PZT transducers. Int J Solids Struct 2002;39(20):5279-93.

[2] Andreaus U, dell'Isola F, Porfiri M. Piezoelectric passive distributed controllers for beam flexural vibrations. J Vibr Control 2004;10(5):625-61.

[3] Canon E, Lenczner M. Modelling of thin elastic plates with small piezoelectric inclusions and distributed electronic circuit. Models for inclusion that are small with respect to the thickness of the plate. J Elast 1999;55:111-41.

[4] Chopra I. Review of state of art of smart structures and integrated systems. AIAA J 2002;40(11):2145-87.

[5] Crandall SH, Karnopp DC, Kurtz Jr EF, Pridmore-Brown FC. Dynamics of mechanical and electromechanical systems. New York: McGraw Hill; 1968. 
[6] Crawley EF, de Luis J. Use of piezoelectric actuators as elements of intelligent structures. AIAA J 1987;25(10): 1373-85.

[7] dell'Isola F, Porfiri M, Vidoli S. Piezo-ElectroMechanical (PEM) structures: passive vibration control using distributed piezoelectric transducers. Compt Rendus l'Acad Sci Mech 2003;331(1):69-76.

[8] dell'Isola F, Maurini C, Porfiri M. Passive damping of beam vibrations through distributed electric networks and piezoelectric transducers: prototype design and experimental validation. Smart Mater Struct 2004;13:299-308.

[9] Fernandes A, Pouget J. An accurate modelling of piezoelectric multi-layer plates. Eur J Mech A/Solids 2002;3(4): 629-51.

[10] Forray MJ. Variational calculus in science and engineering. New York: McGraw Hill; 1968.

[11] Forward RL, Swigert GJ. Electronic damping of orthogonal bending modes in a cylindrical mast-theory. J Spacecraft Rockets 1981;18:5-10.

[12] Hagood NW, von Flotow AH. Damping of structural vibrations with piezoelectric materials and passive electrical networks. J Sound Vibr 1991;146(2):243-368.

[13] Han JH, Rew KH, Lee I. An experimental study of active vibration control of composite structures with a piezoceramic actuator and a piezo-film sensor. Smart Mater Struct 1997;6(5):549-58.

[14] Hanagud S, Obal MW, Calise AJ. Optimal vibration control by the use of piezoceramic sensors and actuators. J Guidance, Control Dyn 1992;15(5):1199-206.

[15] Hoffmann KH, Botkin ND. Homogenization of von Karman plates excited by piezoelectric patches. ZAMM 2000;80(9):579-90.

[16] IEEE Std 176-1987. IEEE Standard on Piezoelectricity. The Institute of Electrical and Electronic Engineers; 1987.

[17] Ikeda T. Fundamentals of piezoelectricity. Oxford: Oxford University Press; 1990.
[18] Jordan TL, Ounaies Z. Piezoelectric ceramics characterization. NASA/CR-2001-211225, CASE Report No. 2001-28.

[19] Kader M, Lenczner M, Mrcarica Z. Distributed control based on distributed electronic circuits: application to vibration control. Microelectr Reliab 2001;41:1857-66.

[20] Kron G. Numerical solutions of ordinary and partial differential equations by means of equivalent circuits. J Appl Phys 1945;16:172-86.

[21] Maurini C, dell'Isola F, Del Vescovo D. Comparison of piezoelectronic networks acting as distributed vibration absorbers. Mech Syst Signal Process 2004;18(5):1243-71.

[22] Maurini C, Pouget J, dell'Isola F. On a model of layered piezoelectric beams including transverse stress effect. Int $\mathrm{J}$ Solids Struct 2004;41(9-10):4473-502.

[23] Molloy CT. Four Pole parameters in vibration analysis in mechanical impedance methods for mechanical vibrations. In: Plunkett R, editor. Colloquium on mechanical impedance methods for mechanical vibrations presented at the ASME annual meeting, New York, NY, December 2, 1958 sponsored by Shock And Vibrating Committee Applied Mechanics Division. New York: The American Society of Mechanical Engineers.

[24] Strikwerda JC. Finite difference schemes and partial differential equations. Pacific Grove, CA: Wadsworth \& Brooks/Cole Advanced Books \& Software; 1989.

[25] Sze KY, Yang XM, Fan H. Electric assumptions for piezoelectric laminate analysis. Int J Solids Struct 2004; 41(9-10):2363-82.

[26] Vidoli S, dell'Isola F. Modal coupling in one-dimensional electro-mechanical structured continua. Acta Mech 2000; 141:37-50.

[27] Vidoli S, dell'Isola F. Vibration control in plates by uniformly distributed actuators interconnected via electric networks. Eur J Mech A/Solids 2001;20:435-56.

[28] Zhou K, Doyle J, Glover K. Robust and optimal control. New Jersey: Prentice-Hall International; 1996. 